\title{
Disentangling surface and bulk photoemission using circularly polarized light
}

\author{
V. B. Zabolotnyy, ${ }^{1}$ S. V. Borisenko, ${ }^{1}$ A. A. Kordyuk,${ }^{1,2}$ D. S. Inosov, ${ }^{1}$ A. Koitzsch, ${ }^{1}$ J. Geck, ${ }^{1}$ J. Fink,,${ }^{1}$ M. Knupfer, \\ B. Büchner, ${ }^{1}$ S.-L. Drechsler, ${ }^{1}$ V. Hinkov, ${ }^{3}$ B. Keimer, ${ }^{3}$ and L. Patthey ${ }^{4}$ \\ ${ }^{1}$ Institute for Solid State Research, IFW-Dresden, P.O.Box 270116, D-01171 Dresden, Germany \\ ${ }^{2}$ Institute of Metal Physics of National Academy of Sciences of Ukraine, 03142 Kyiv, Ukraine \\ ${ }^{3}$ Max-Planck Institut für Festkörperforschung, D-70569 Stuttgart, Germany \\ ${ }^{4}$ Swiss Light Source, Paul Scherrer Institut, CH-5234 Villigen, Switzerland
}

(Received 21 February 2007; published 5 July 2007)

\begin{abstract}
We show that in angle resolved photoemission spectroscopy (ARPES), near-surface induced fields can be useful for disentangling the surface and bulk related emission. The jump of the dielectric function at the interface results in a nonzero term $\operatorname{div} \mathbf{A}$ in the photoemission matrix element. The term happens to be significant approximately within the first unit cell and leads to circular dichroism for the states localized therein. As an example we use ARPES spectra of an $\mathrm{YBa}_{2} \mathrm{Cu}_{3} \mathrm{O}_{7-\delta}$ crystal to distinguish between the overdoped surface related component and its bulk counterparts.
\end{abstract}

DOI: 10.1103/PhysRevB.76.024502

PACS number(s): 74.25.Jb, 74.72.Hs, 79.60.-i

Angle resolved photoelectron spectroscopy (ARPES) is subjected to a known limitation, which is the surface sensitivity caused by a rather small escape depth of emitted photoelectrons. According to the universal curve ${ }^{1}$ the mean free path for the photo electrons in the energy range of $20-70 \mathrm{eV}$ is about 5-10 $\AA$, which is comparable to the unit cell of the most of the studied materials. In numerous photoemission studies it is frequently assumed that the spectra from the first unit cell already reflect the bulk properties of the material and that the surface related effects are of minor influence. However this is not always the case. ARPES spectra of the high temperature superconductor $\mathrm{YBa}_{2} \mathrm{Cu}_{3} \mathrm{O}_{7-\delta}$ are just one of the examples. ${ }^{2,3}$ The signal picked up from the near surface region corresponds to an unusually high hole doping level and displays no superconductivity, while in the bulk the samples are characterized by a narrow superconducting transition and uniform doping level, which clearly shows that the bulk and surface have different properties. Possible way to enhance the bulk contribution consist in the use of comparatively small $(5-10 \mathrm{eV})$ (Ref. 4) or large $(\sim 1000 \mathrm{eV})$ (Ref. 5) excitation energies. However, both of these approaches have certain restrictions. In the first case it becomes impossible to probe the states in the whole Brillouine zone, as $k_{\| \max }=\sqrt{2 m E_{\mathrm{kin}}} / \hbar$ becomes too small, while in the high energy case significant deterioration of the momentum resolution takes place, not to mention that both approaches require a specialized light source, which might not always be available.

The fact that the electron escape depth equals $\lambda$ only means that the electron intensity is attenuated by the factor $\exp (-z / \lambda)$, i.e., there are still photoelectrons leaving the solid from the depth $z>\lambda$, but their intensity is decreasing according to the exponential law. Therefore the spectra do contain a signal reflecting the bulk properties, but the problem is how to extract it from under the bright surface contribution. Fortunately the bulk and surface photoemission differ also due to the pattern of the electromagnetic field that excites the electrons and this can be used as a "marker" that makes these two spectral components discernable. Already in studies of $\mathrm{Cu}$ surface states it was shown that the photoemission matrix elements are strongly modified by the term $\operatorname{div} \mathbf{A}$, which becomes important at the near surface region due to the mismatch in the dielectric constant between the solid and the vacuum, and the calculated dependence of the matrix element on the incidence angle of the linearly polarized exciting radiation was found to be in a good agreement with experimental data. ${ }^{6}$ The depth down to which the term div A modifies the matrix elements depends on the field pattern near the surface, the exact microscopic calculation of which turns out to be a very difficult task. Nevertheless, a basic insight can be obtained considering a relatively simple jellium model. Employed to describe a free electron metal, ${ }^{7}$ the model shows that the vector potential of the electromagnetic wave $\mathbf{A}(\mathbf{r})$ experiences quickly decaying Friedel-like oscillations with a characteristic scale of a few angstroms. In a more recent study of $\mathrm{TiS}_{2}$, which is closer to the case we are going to concentrate later on, the layered structure of the crystal was taken into account. ${ }^{8}$ From this study follows that rapid changes that $\mathbf{A}(\mathbf{r})$ undergoes are happening within thin surface layer with characteristic thickness $d_{\text {surf }}$ of about one unit cell along the normal to the surface, which is qualitatively a general picture that would follow from any microscopic model.

In this manuscript we show that the discussed term $\operatorname{div} \mathbf{A}(\mathbf{r})$ leads to a circular dichroism for the photoemission from the surface layer and how the dichroism can be used to obtain information about the states in the bulk and at the surface.

In general, the photoemission matrix elements depend on experimental geometry (i.e., mutual position of the sample, polarization, wave vector of incident light $\mathbf{q}$ and the direction of emitted photoelectron k). In Fig. 1. we depict the geometry of our experiment. The entrance slit of the energy analyzer is vertical and its position is fixed in space, so that the ARPES spectrum, the so called energy-momentum distribution, is just a 2D distribution of photointensity as a function of energy and the angle $\eta$ at which electrons enter the analyzer entrance slit. For a fixed sample position the wave vector $\mathbf{k}$ of the photoelectron and its projections $k_{x}$ and $k_{y}$ on the sample surface are uniquely defined by the angle $\eta$ and can be easily estimated from the drawing. The incident beam lies in the horizontal plane $M O N$ with $\angle M O N \equiv \beta=45^{\circ}$. Differ- 

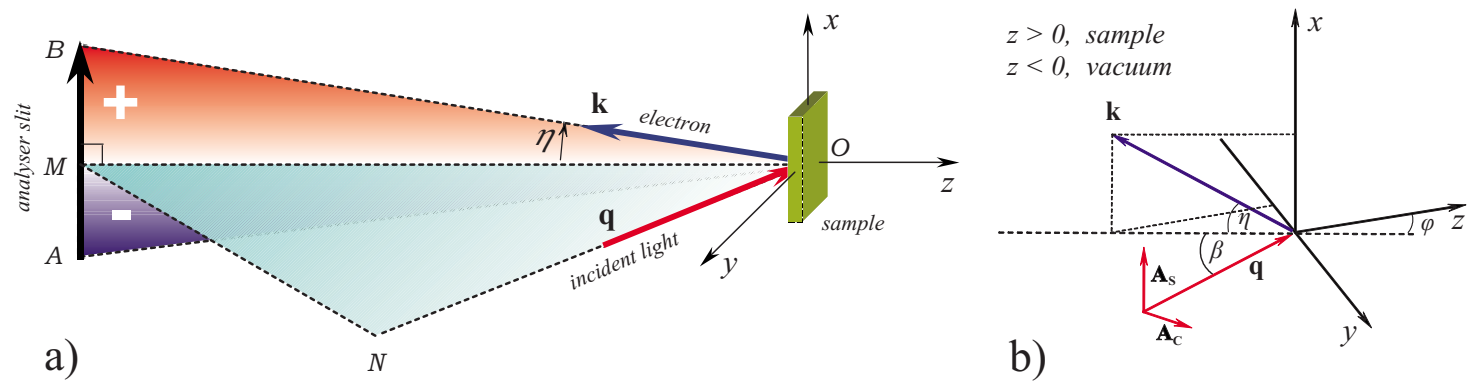

b)

FIG. 1. (Color online) Experimental geometry. (a) Linear dependence of the circular dichroism in photoelectron intensity $D=I^{\mathrm{cr}}-I^{\mathrm{cl}}$ on the angle $\eta$. The red-white-blue color scale denotes the strength of the dichroism. (b) Decomposition of a circularly polarized wave in two linearly polarized ones with polarization vectors $\mathbf{A}_{\mathrm{S}}$ and $\mathbf{A}_{\mathrm{C}}$ with a phase shift of $\pi / 2$.

ent parts of reciprocal space can be probed via rotation of the sample around the pivot point $O$. For instance in Fig. 1(b) we show the sample (the crystal primary axes) after rotation around the $x$ axis by the angle $\varphi$, when the projection $k_{y}$ becomes negative in contrast to Fig. 1(a), where $\varphi=0$ and $k_{y}=0$.

The photoemission matrix element can be estimated as a probability of a transition between the initial state $|i\rangle$ and the final state $|\mathrm{f}\rangle$, which is given by the Fermi golden rule: ${ }^{9}$

$$
w_{\mathrm{i} \rightarrow \mathrm{f}} \sim \frac{2 \pi}{\hbar}\left|\left\langle\mathrm{f}\left|\hat{H}_{\text {pert }}\right| \mathrm{i}\right\rangle\right|^{2} \delta\left(E_{\mathrm{f}}-E_{\mathrm{i}}-\hbar \omega\right),
$$

where the perturbation to the system Hamiltonian $\hat{H}_{\text {pert }}=$ $-\frac{e i \hbar}{m c}\left(\mathbf{A} \boldsymbol{\nabla}+\frac{1}{2} \operatorname{div} \mathbf{A}\right)$. The incident circularly polarized wave $\mathbf{A}(\mathbf{r})$ can be represented as a sum of two plane waves [Fig. 1(b)]

$$
\mathbf{A}(\mathbf{r})=\mathbf{A}_{\mathrm{C}} \cos (\omega t-\mathbf{q} \cdot \mathbf{r})+\mathbf{A}_{\mathrm{S}} \sin (\omega t-\mathbf{q} \cdot \mathbf{r}) .
$$

It is easy to show that for such a periodic perturbation

$$
\begin{aligned}
& w_{\mathrm{i} \rightarrow \mathrm{f}} \sim \frac{2 \pi}{\hbar}\left|\left\langle\mathrm{f}\left|\hat{V}_{\mathrm{C}}-i \hat{V}_{\mathrm{S}}\right| \mathrm{i}\right\rangle\right|^{2}, \quad \text { where } \\
& \hat{V}_{\mathrm{C}, \mathrm{S}}=-\frac{e i \hbar}{m c}\left(\mathbf{A}_{\mathrm{C}, \mathrm{S}} \boldsymbol{\nabla}+\frac{1}{2} \operatorname{div} \mathbf{A}_{\mathrm{C}, \mathrm{S}}\right) .
\end{aligned}
$$

At this stage the particular pattern of the near surface induced field comes into play. Macroscopically, from the continuity condition for the components of $\mathbf{D}$ and $\mathbf{E}$ vectors one has

$$
\begin{gathered}
\mathbf{E}_{\|}^{\text {vacuum }}=\mathbf{E}_{\|}^{\text {sample }}, \\
\mathbf{E}_{\perp}^{\text {vacuum }}=\mathbf{D}_{\perp}^{\text {vacuum }}=\mathbf{D}_{\perp}^{\text {sample }}=\varepsilon \mathbf{E}_{\perp}^{\text {sample }},
\end{gathered}
$$

where subscripts $\|(\perp)$ indicate vector components parallel (perpendicular) to the sample surface, and $\varepsilon$ is the sample permeability. Using the gauge with the scalar potential of the electromagnetic field taken to be zero, the last equation can be rewritten as

$$
\mathbf{A}_{\|}^{\text {vacuum }}=\mathbf{A}_{\|}^{\text {sample }}, \quad \mathbf{A}_{\perp}^{\text {vacuum }}=\varepsilon \mathbf{A}_{\perp}^{\text {sample }} .
$$

Microscopically, as has previously been pointed out, the jump in the vector potential appears as a smooth transition with a spatial extension $d_{\text {surf }}$ of about one lattice parameter $c$, within which the vector potential $\mathbf{A}(\mathbf{r})$ changes from its vacuum value to the bulk one. For the discussed geometry $\operatorname{div} \mathbf{A}_{\mathrm{S}}=0$ due to the boundary conditions, and $\operatorname{div} \mathbf{A}_{\mathrm{C}}$ can be approximated as

$$
\begin{aligned}
\operatorname{div} \mathbf{A}_{C} & \approx \frac{\left|\mathbf{A}_{\perp}^{\text {sample }}-\mathbf{A}_{\perp}^{\text {vacuum }}\right|}{d_{\text {surf }}}=\frac{(1 / \varepsilon-1)\left|\mathbf{A}_{\perp}^{\text {vacuum }}\right|}{d_{\text {surf }}} \\
& =C\left|\mathbf{A}_{\perp}^{\text {vacuum }}\right|=C\left|\mathbf{A}_{C}\right| \sin (\beta-\varphi),
\end{aligned}
$$

where $C$ is a complex constant that effectively accounts for the width of the transition layer and sample permeability.

To estimate the matrix element (3) we assume that the final state is a plane wave: $|\mathrm{f}\rangle=\left|e^{i \mathbf{k} \cdot \mathbf{r}}\right\rangle$, with $\mathbf{k}$ being the quasimomentum of the exited photoelectron. Leaving out the irrelevant coefficients yields

$$
\begin{aligned}
w_{\mathrm{i} \rightarrow \mathrm{f}} & \sim\left|\left\langle\mathrm{i}\left|\mathbf{A}_{\mathrm{C}} \boldsymbol{\nabla}+\frac{1}{2} \operatorname{div} \mathbf{A}_{\mathrm{C}}-i \mathbf{A}_{\mathrm{S}} \boldsymbol{\nabla}\right| e^{i \mathbf{k} \cdot \mathbf{r}}\right\rangle\right|^{2} \\
& =\left|\left\langle\mathrm{i} \mid e^{i \mathbf{k} \cdot \mathbf{r}}\right\rangle\right|^{2}\left|\mathbf{A}_{\mathrm{C}} i \mathbf{k}+\mathbf{A}_{\mathrm{S}} \mathbf{k}+\frac{1}{2} \operatorname{div} \mathbf{A}_{\mathrm{C}}\right|^{2} .
\end{aligned}
$$

To get the matrix element for the opposite circular polarization one just needs to reverse the direction of the vector $\mathbf{A}_{S}$, therefore for the dichroism we obtain

$$
\begin{aligned}
D \equiv & \mathrm{d} w_{\mathrm{i} \rightarrow \mathrm{f}}^{\mathrm{cr}}-\mathrm{d} w_{\mathrm{i} \rightarrow \mathrm{f}}^{\mathrm{cl}} \\
\sim & \left|\left\langle\mathrm{i} \mid e^{i \mathbf{k} \cdot \mathbf{r}}\right\rangle\right|^{2}\left\{\left|\mathbf{A}_{\mathrm{C}} i \mathbf{k}+\mathbf{A}_{\mathrm{S}} \cdot \mathbf{k}+\frac{1}{2} \operatorname{div} \mathbf{A}_{\mathrm{C}}\right|^{2}\right. \\
& \left.-\left|\mathbf{A}_{\mathrm{C}} i \mathbf{k}-\mathbf{A}_{\mathrm{S}} \cdot \mathbf{k}+\frac{1}{2} \operatorname{div} \mathbf{A}_{\mathrm{C}}\right|^{2}\right\} .
\end{aligned}
$$

Since $\left|\mathbf{A}_{C} i \mathbf{k}-\mathbf{A}_{\mathrm{S}} \cdot \mathbf{k}\right|=\left|\mathbf{A}_{\mathrm{C}} i \mathbf{k}+\mathbf{A}_{\mathrm{S}} \cdot \mathbf{k}\right|$ there will be no circular dichroism if $\operatorname{div} \mathbf{A}_{\mathrm{C}}$ equals zero. It is worth to mention that this is a rather general statement true for any geometry as long as the final states can be well approximated by the plane waves. ${ }^{10}$ As pointed out in Ref. 11 the deviation of the final states from plane waves might be considerable for low kinetic energies $(\lessgtr 10 \mathrm{eV})$, hence leading to possible circular dichroism. Nonetheless, for the energy range that we are using the free electron approximation is well justified ${ }^{12,13}$ and the dichroism due to nonplane wave character of final states can be excluded. 

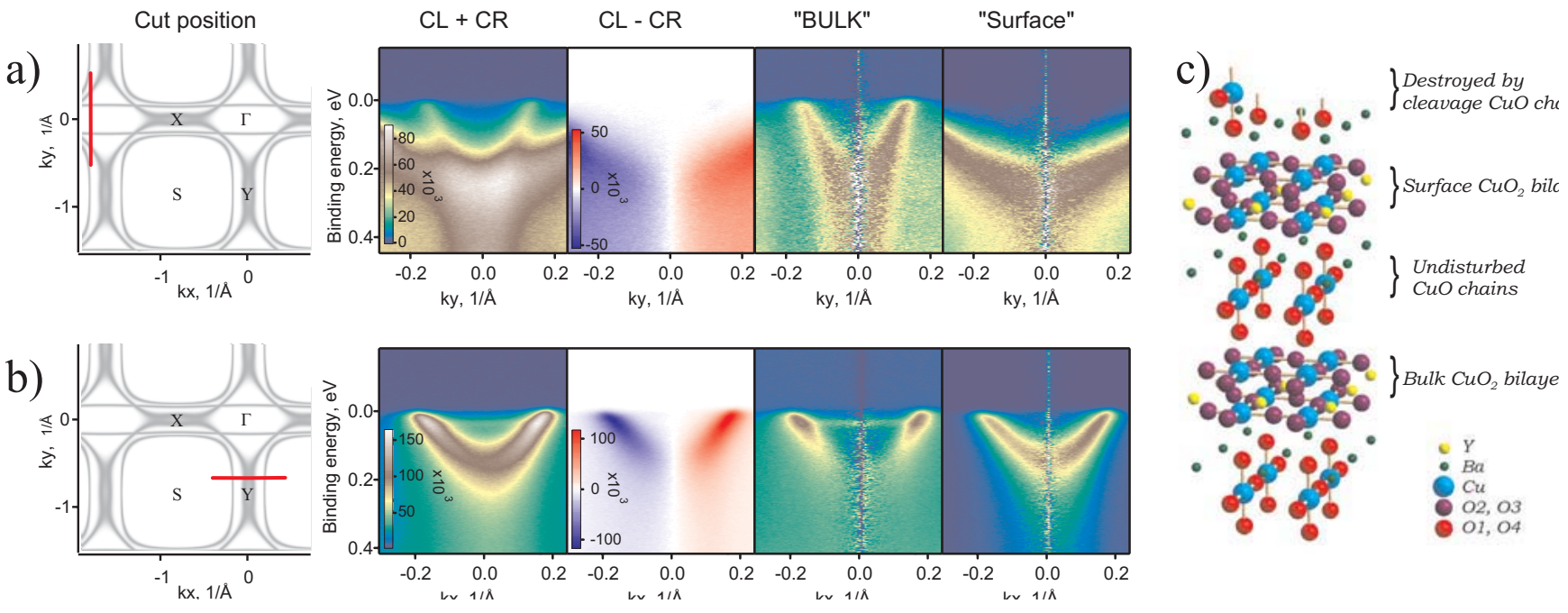

FIG. 2. (Color online) (a), (b) Left to right: position of the energy-momentum cuts in the reciprocal space, sum of the spectra measured with opposite circular polarizations, their difference, extracted bulk component, extracted surface component. Color scale bars show the absolute intensity. In the block (a) the process of disentangling the surface related antibonding band and the chain band is demonstrated $\left(h \nu=50 \mathrm{eV}, k_{x}^{\text {slit }} \approx-1.82 \AA^{-1}\right)$. (b) The same procedure, but for the surface and bulk components of the bonding band $\left(h \nu=55 \mathrm{eV}, k_{y}^{\text {slit }}\right.$ $\approx-0.70 \AA^{-1}$ ). The antibonding band in this case is suppressed by the unfavorable matrix elements. (c) $\mathrm{Structure}$ of $\mathrm{YBa}_{2} \mathrm{Cu}_{3} \mathrm{O}_{7-\delta}$ near the cleavage plane.

After simple computations, taking into account the experimental geometry, the expression (8) reduces to the final form that we are going to use

$$
D \sim\left|\left\langle\mathrm{i} \mid e^{i \mathbf{k} \cdot \mathbf{r}}\right\rangle\right|^{2} \operatorname{Re}(C)\left|\mathbf{A}_{\mathrm{C}} \| \mathbf{A}_{\mathrm{S}}\right||\mathbf{k}| \sin (\eta) \sin (\beta-\varphi) .
$$

An important consequence of this formula is that in the described geometry the photoemission signal arising from the near surface region would exhibit circular dichroism proportional to $\sin (\eta) \approx \eta$. It is this particularity that can be used to distinguish the contribution to the spectrum arising from the near surface layer, where the term with $\operatorname{div} \mathbf{A}$ is important, from the one coming from the deeper regions of the sample. Representing two-dimensional ARPES spectra as a sum of a "bulk" and the "surface" component we can write

$$
\begin{aligned}
& I^{\mathrm{cl}}(\eta, \omega)=I^{\text {bulk }}(\eta, \omega)+I^{\text {surf }}(\eta, \omega)(1+\alpha \eta), \\
& I^{\mathrm{cr}}(\eta, \omega)=I^{\text {bulk }}(\eta, \omega)+I^{\text {surf }}(\eta, \omega)(1-\alpha \eta),
\end{aligned}
$$

where $\alpha$ accounts for the dichroism strength. The constituent bulk and surface related components can easily be obtained:

$$
\begin{gathered}
I^{\mathrm{surf}}(\eta, \omega)=\frac{1}{2 \alpha \eta}\left[I^{\mathrm{cl}}(\eta, \omega)-I^{\mathrm{cr}}(\eta, \omega)\right], \\
I^{\mathrm{bulk}}(\eta, \omega)=\frac{1}{2}\left[I^{\mathrm{cl}}(\eta, \omega)+I^{\mathrm{cr}}(\eta, \omega)\right]-I^{\mathrm{surf}}(\eta, \omega) .
\end{gathered}
$$

Now we proceed to the practical application of the derived formulas using as an example ARPES spectra of $\mathrm{YBa}_{2} \mathrm{Cu}_{3} \mathrm{O}_{7-\delta}$ (Fig. 2). The spectra were measured from a freshly cleaved surfaces at $T=30 \mathrm{~K}$ with SES100 SCIENTA electron energy analyzer at the SIS beam line at Paul Scherrer Institute. The energy and angular resolution were $15 \mathrm{meV}$ and $0.2^{\circ}$, respectively. The excitation energy is given in the caption to the figure, further experimental details can be found elsewhere. ${ }^{14}$

Similar to many other layered compounds its weak $k_{z}$ dispersion allows for a relatively simple exposition of ARPES data. In this case the spectral function depends only on three parameters, so its argument can be thought of as a point in the three-dimensional (3D) space spanned over one energy and two momentum axes. Therefore the 2D energymomentum intensity distributions, already mentioned during the discussion of experimental geometry, are practically almost plane cuts through this $3 \mathrm{D}$ space with the bright features therein corresponding to the traces of the renormalized electronic bands. ${ }^{15}$ In the leftmost panels of Figs. 2(a) and 2(b) we show a model of the $\mathrm{YBa}_{2} \mathrm{Cu}_{3} \mathrm{O}_{7-\delta}$ Fermi surface map that consists of pairs of squarelike contours around the $\mathrm{S}$ points, corresponding to the bonding and antibonding bands, and features parallel to $k_{x}$ axis, which are the Fermi level crossings of the chain derived band. The red segments denote the position in the $k$ space of the momentum-energy distribution given in the next panels, which are: the sum of spectra obtained with the opposite circular polarizations; the difference of the spectra; the extracted bulk and surface contributions based on formulae (11). To achieve maximal subtraction of the surface component, parameter $\alpha$ was increased until negative values appeared.

From the Fig. 2(a) it follows that the states of the intense overdoped antibonding band are supposed to be localized at the near surface region, while the chain band has to be of bulk origin as it displays no dichroism. To understand this one needs to look in detail at the structure of cleaved $\mathrm{YBa}_{2} \mathrm{Cu}_{3} \mathrm{O}_{7-\delta}$ shown in Fig. 2(c). According to the tunneling experiments ${ }^{16,17}$ this crystal cleaves between $\mathrm{BaO}$ and $\mathrm{CuO}$ layers, which is schematically shown in the figure as broken bonds and missing atoms. The remnants of the $\mathrm{CuO}$ chains, heavily disrupted by the cleavage, are unlikely to result in 
photoemission signal that reminds the bulk dispersion of this band. Therefore the signal from the nearest to the surface $\mathrm{CuO}_{2}$ bilayer should be the brightest. In a view of missing chain structure on top of this bilayer, the change of its hole doping level is not surprising. ${ }^{2,3}$ The absence of the dichroism for the chain states simply means that the nearest to the surface chains are already out of the region where $\mathbf{A}(\mathbf{r})$ strongly oscillates resulting in perceivable $\operatorname{div} \mathbf{A}$. This also means that the next $\mathrm{CuO}_{2}$ bilayer, which we expect to be superconducting as it is surrounded by the $\mathrm{CuO}$ chains on its both sides, resides in the region, where $\operatorname{div} \mathbf{A}$ becomes negligible and should exhibit no dichroism, similar to the chain band. Indeed, splitting the spectrum of the bonding band [Fig. 2(b)] into surface and bulk contributions we see that the surface component reminds the spectrum of the normal state taken above $T_{\mathrm{C}}$, exhibiting no unusual renormalization. The spectrum of the bulk component looks qualitatively different. The strong band renormalization, which is a known signature of the superconducting state, ${ }^{18-22}$ is clearly visible in the spectrum, supporting the expectation.

Aiming at a true quantitative analysis of the disentangled spectra one needs to be cautious as the dichroism in photoemission experiments is a rather ubiquitous phenomenon. The magnetic dichroism could be a one possible reason, but it rarely exceeds $1-3 \%,{ }^{23}$ which is much less than the strength $\left(I^{\mathrm{cr}}-I^{\mathrm{cl}}\right) /\left(I^{\mathrm{cr}}+I^{\mathrm{cl}}\right)$ of the described surface related dichroism that exceeds $60 \%$. One may expect a dichroism for the case when the degeneracy of the valence bands is lifted by the spin-orbit interaction, as in the case of noncentrosymmetric superconductor $\mathrm{CePt}_{3} \mathrm{Si}^{24} \mathrm{Such}$ a possibility was investigated earlier in Ref. 25. The relativistic calculation showed that the spin-orbit coupling could potentially result in the effect in the case of $\mathrm{Bi}-2212$ cuprate, but only due to the fact that it has a high- $Z \mathrm{Bi}$ in its structure. There- fore, we exclude spin-orbit interaction as a possible reason for dichroism in Y-123.

Dichroism can also arise from the bulk states. ${ }^{26}$ While the surface related dichroism vanishes at the normal light incidence [Eq. (9), $\beta=\varphi$ ], the bulk one is expected to be still nonvanishing if the experimental setup (including the sample) possesses definite handedness and the final states substantially differ from the plane waves. To estimate its strength in cuprates we can refer to our previous results, ${ }^{27}$ where the total dichroism for the normal light incidence was shown to be not more than 6\%. For the arbitrary light incidence $^{25}$ (when the surface dichroism is vanishing due to condition $\eta=0$ ) the total dichroism amounts to $\sim 18 \%$, but for the sample orientation discussed here, i.e., when one of the crystal primary axes lies in the $M O N$ plane, its value is zero again, so that the surface related dichroism turns out to be a dominating one.

To conclude, we have stressed the importance of the near surface induced electromagnetic fields for the interpretation of photoemission data, and pointed out that those might be a beneficial factor allowing for distinguishing between the surface and bulk photoemission. In some cases it is also possible to localize the position of the surface and bulk states more precisely, as in the case of $\mathrm{YBa}_{2} \mathrm{Cu}_{3} \mathrm{O}_{7-\delta}$, where the nearest to the surface $\mathrm{CuO}_{2}$ bilayer happens to be overdoped, while the next bilayer can already be treated as the bulk one. The obtained results can easily be extended to any other experimental geometry.

The project is part of the Forschergruppe FOR538 and was supported by the DFG under Grant No. KN393/4 and by BMBF under Grant No. 05KS40D218. The measurements were done at SIS 9L beamline, Swiss Light Source, Paul Scherrer, Villigen, Switzerland. We thank R. Hübel for technical support.
${ }^{1}$ M. Seah and W. Dench, Surf. Interface Anal. 1, 2 (1979).

${ }^{2}$ V. B. Zabolotnyy et al., arXiv:cond-mat/0608295 (to be published).

${ }^{3}$ K. Nakayama et al., arXiv:cond-mat/0611515 (to be published).

${ }^{4}$ J. D. Koralek et al., Phys. Rev. Lett. 96, 017005 (2006).

${ }^{5}$ S. Suga et al., Phys. Rev. B 70, 155106 (2004).

${ }^{6}$ F. Pforte, T. Michalke, A. Gerlach, A. Goldmann, and R. Matzdorf, Phys. Rev. B 63, 115405 (2001).

${ }^{7}$ P. J. Feibelman et al., Phys. Rev. Lett. 34, 1092 (1975).

${ }^{8}$ D. Samuelsen and W. Schattke, Phys. Rev. B 51, 2537 (1995).

${ }^{9}$ L. Landau and E. Lifshitz, Quantum Mechanics, Non-Relativistic Theory (Pergamon, Oxford, 1977).

${ }^{10}$ R. L. Dubs, S. N. Dixit, and V. McKoy, Phys. Rev. B 32, 8389 (1985).

${ }^{11}$ V. N. Strocov et al., Phys. Rev. Lett. 81, 4943 (1998).

${ }^{12}$ A. Baalmann, M. Neumann, W. Braun, and W. Radlik, Solid State Commun. 54, 583 (1985).

${ }^{13}$ J. Olde, G. Mante, H. P. Barncheidt, L. Kipp, J.-C. Kuhr, R. Manzke, M. Skibowski, J. Henk, and W. Schattke, Phys. Rev. B 41, 9958 (1990).

${ }^{14}$ S. V. Borisenko et al., Phys. Rev. Lett. 96, 117004 (2006).

${ }^{15}$ S. V. Borisenko et al., Phys. Rev. B 64, 094513 (2001).
${ }^{16}$ H. L. Edwards, J. T. Markert, and A. L. deLozanne, Phys. Rev. Lett. 69, 2967 (1992).

${ }^{17}$ S. H. Pan et al., Rev. Sci. Instrum. 70, 1459 (1999).

${ }^{18}$ A. Kaminski, M. Randeria, J. C. Campuzano, M. R. Norman, H. Fretwell, J. Mesot, T. Sato, T. Takahashi, and K. Kadowaki, Phys. Rev. Lett. 86, 1070 (2001).

${ }^{19}$ P. V. Bogdanov et al., Phys. Rev. Lett. 85, 2581 (2000).

${ }^{20}$ T. K. Kim, A. A. Kordyuk, S. V. Borisenko, A. Koitzsch, M. Knupfer, H. Berger, and J. Fink, Phys. Rev. Lett. 91, 167002 (2003).

${ }^{21}$ Ar. Abanov, A. V. Chubukov, Phys. Rev. Lett. 83, 1652 (1998).

${ }^{22}$ M. Eschrig and M. R. Norman, Phys. Rev. B 67, 144503 (2003).

${ }^{23}$ B. Ritchie et al., Phys. Rev. A 12, 567 (1975).

${ }^{24}$ K. V. Samokhin, E. S. Zijlstra, and S. K. Bose, Phys. Rev. B 69, 094514 (2004).

${ }^{25}$ S. V. Borisenko et al., Phys. Rev. B 69, 224509 (2004).

${ }^{26}$ T. Matsushita, S. Imada, H. Daimon, T. Okuda, K. Yamaguchi, H. Miyagi, and S. Suga, Phys. Rev. B 56, 7687 (1997).

${ }^{27}$ S. V. Borisenko, A. A. Kordyuk, A. Koitzsch, T. K. Kim, K. A. Nenkov, M. Knupfer, J. Fink, C. Grazioli, S. Turchini, and H. Berger, Phys. Rev. Lett. 92, 207001 (2004). 